\begin{tabular}{|c|c|}
\hline \multirow{3}{*}{ 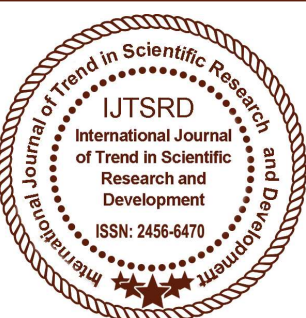 } & $\begin{array}{l}\text { International Journal of Trend in Scientific } \\
\text { Research and Development (IJTSRD) }\end{array}$ \\
\hline & Open Access Journal \\
\hline & ISSN No: 2456 - 6470 | www.ijtsrd.com | Volume - 2 | Issue -3 \\
\hline
\end{tabular}

\title{
Review Paper on Biomedical Image processing using Wavelets
}

\author{
Miss. Nishigandha M. Sawarkar \\ Department of Electronics and Telecommunication \\ Engineering, Sipna college of Engineering and \\ Technology Amravati, India
}

\author{
Prof. S. S. Mungona \\ Department of Electronics and Telecommunication \\ Engineering, Sipna college of Engineering and \\ Technology Amravati, India
}

\begin{abstract}
One of the most common problem in human being is bone fracture. Bone fracture can occur due to accident or due to high pressure applied on bone. Another reason to occur bone fracture can be osteoporosis and bone cancer. Therefor accurate diagnosis is important. The fracture can occur in any bone of our body like wrist, heel, ankle, rib, leg, hib, chest, etc. the fracture can not detect by naked eyes. To detect it $\mathrm{X}$-rays and CT images are used. Now a days image processing plays an important role in modern data storage. Image processing is important in modern data storage and transmission. This paper presents a study of image processing techniques for bone fracture detection. This paper will help user to design new techniques to improve accuracy of fracture detection. This project employed MATLAB for loading image, image processing as a programming tool.
\end{abstract}

Keywords: X-rays; CT images; osteoporosis.

\section{Introduction}

The facture can be defined as crack in bone or break in a bone. Proteins, fibers and minerals combined to form a bone of our body. Number of bones are joined together to form a skeleton of the body. Support to the body shape, protection to the organ of body is provided by skeleton. Bones helped to produce red blood cells. With help of bones we can walk, seat, run, jump, kneel, lift. Bones also protect our internal organs from the damage. Various shape, size and structure of bone occurs in human body. Femure bone are the largest bones and auditory bones are the smallest bones. Different types of bone fractures occurs as oblique, compound, spiral, greenstick and transverse. To identify such a different types bone fractures different medical imaging tools are used such as X-rays, CT(computed tomography) images, MRI(Magnetic Resonance Imaging), ultrasound, etc. Most commonly used technique are X-rays and CT images in fracture detection. These techniques provide easiest way to diagnosis. For existence and location of fracture, doctors usually use X-rays. In the children, broken bones are very common. In children, the fractures are easily recovered than the adults. The broken bones can recover within 28 to 56 days and also it may depend on the person age and health and type of fracture. A fracture can be crosswise, lengthwise, in several places, or into small or two or more pieces. Typically, a bone fracture can be occurred by the force or pressure or fall from vehicles etc. The input image is taken from the user. The input image can be in any form like X-rays, skin images, eye images etc. These images then forwarded to image processing block. Image processing includes image feature extraction using wavelets. The wavelets can be used for image enhancement and image compression. It can be extract different features of images. Pixel classification can be done on the basis of intensity of pixels. In X-rays, there are three types of different intensities can be occur such as background, bones, and fractured region in X-rays. In this region we can identified the fracture region using pixel classifications. The next block is Image segmentation which is used to detect the boundaries of an image. In this section we can determine the actual length of the fracture. The calculated result will be applied with fuzzy logics. To apply fuzzy logics there are different fuzzy rules by which we can 
calculate the actual result. The obtained result will be analyzed with the previous observations. In this way we can obtain the different result for different images as input taken into considerations.

\section{Literature Review}

In this section different method applied to $\mathrm{x}$-ray/CT images are listed and corresponding papers were discussed. This will help the reader in understanding the potential and amount of research that have been carried in that field. An attempt has been made in providing short technical details of each paper, for the benefit of researchers in this field. This paper also presents some common and new tools used for image processing in the study of bone fracture detection. Normally Classification based and transformed base are two types of fracture detection technique. This paper discusses both the techniques. Wavelet and Curvelet, Haar Chan, K.P [1] proposed a method of feature selection by using three different methods such as wavelet and curvelets transform. Haar method gives the highest accuracy value compared with other two methods.

Support Vector Machine(SVM) classifier Lim, S. E at al [2], Yap, D. at al [3] and Lum, V. L. F at [4] proposed to use Gabor, Markov Random Field, and gradient intensity features extracted from the x-ray images and fed into Support Vector Machines (SVM) classifiers. They observe that the combination of three SVM classifiers improves the overall accuracy and sensitivity compared to using individual classifiers.

Generally the DICOM images are corrupted by the salt and pepper noise. Al-Khaffaf $\mathrm{H}$ at al [5] proposed an extension of the K-fill algorithm to remove salt and pepper noise based on the number of black or white pixels in a $3 \times 3$ window. Assuming that the images are corrupted by the noise modeled as a sum of two random processes: a Poisson and a Gaussian, this approach allows them to jointly estimate the scale parameter of the Poisson component and the mean and variance of the Gaussian one.

Vijaykumar V[6] presented a filtering algorithm for Gaussian noise removal. First estimating the amount of noise from the noisy image, then replace the center pixel by the mean of the sum of the surrounding pixels based on a threshold value. Compared to other filtering algorithms such as mean, alpha-trimmed mean, Wiener, K-means, bilateral and trilateral, this algorithm gives lower Mean Absolute Error (MAE) and higher Peak Signal-to-Noise Ratio (PSNR).

Active contour model (ACM and GACM) R. Aishwariya, Geeta, M.Kalaiselvi [7] proposed the technique that detect the boundaries of objects in noisy images using the information the fracture detection on the $\mathrm{x}$-ray images is founded. The proposed technique for the canny edge detector in the x-ray image locates the edges and using the boundary detection, the system which detects the fracture automatically. The boundary detection techniques also implemented in the models are Active Contour Model, Geodesic Active Contour Model and compare the accuracy of detecting is analyzed and tested Using Matlab.

X-Ray/CT auto classification of fracture (GLCM) Anu T C, Mallikarjunaswamy M.S Rajesh Raman[8] proposed computer based analysis techniques for the detection of bone fracture using X-ray/CT images. It starts from the preprocessing to remove the noise and edge detected by using sobel edge detector. After the segmentation the area of the fracture is calculated. The method has been tested on a set of images and results have been evaluated based on GLCM features. Analysis shown that results obtained are satisfactory and accuracy of this method was $85 \%$.The limitation of this method is , in CT and some cases of X-ray images very difficult to find the area of fracture.

Gray-Level Co-occurrence Matrix (GLCM) is used for feature extraction and selection. GLCM was defined by Haralick..GLCM is main tool used in image texture analysis. Textures of an image are complex visual patterns that are composed of entities or regions with subpatterns with the characteristics of brightness, color, shape, size, etc. GLCM is a statistical way to indicate image texture structure by statistically sampling the pattern of the grey-levels occurs in relation to other grey levels.

Swathika.B, Anandhanarayanan.K, Baskaran.B, and Govindaraj.R [9] proposed Novel morphological gradient based edge detection technique in which canny edge detection is applied after finding morphology gradient. The morphological gradient technique removes noise, enhances image details and highlights the fracture region. The fracture edges are more prominently revealed due to the combined effect of morphological gradient technique and 
canny edge detection algorithm. The processed image output show that the proposed technique provides efficient fracture detection when compared with other edge detection methods. Using GLCM and fcm Hs Rathode and Wahid Ali [10] proposed an algorithm for Automatic tumor detection that is based on segmentation using Daubechies Wavelet and Fuzzy C-Means (FCM) Clustering. The segmented portion showing the tumor area in pixels and the time elapsed to detect and calculate the area in seconds.

Tanudeep Kaur , Anupam Garg [11] proposed wavelet approach used to detect Fracture detection region on the X-Ray images. Multilevel wavelet is used to find the fracture from the X-ray bone images after applying FCM and Gabor filter. It finds fracture only in horizontal images. It uses Hough transform to find long bones and wavelet decomposition. It finds the fracture only the horizontal images.

\section{System Architecture}

\section{A. Block Diagram}

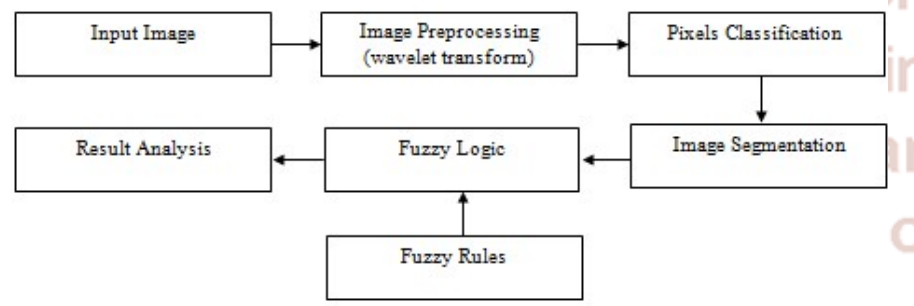

\section{Description :}

The X-Rays /CT images are obtained from the hospital that contains normal as well as fractured bone images. In the first step applying preprocessing techniques such as RGB to gray scale conversion. The image enhancement can be obtained by removing noise from the image. Then image classification can be done so as to separate background, ROI(region of interest) and infected pixels. Then infected pixels get marked. Then this images will be compared with the given data base and result is calculated by applying fuzzy logic to it. The block description can be given as below.

Input Image: The input image can be obtained from the patient or from the hospital in the form of X-rays or CT images.

Image Preprocessing: In computer aided diagnosis of medical images, noise removal and image enhancement are the tool for image processing. The noise can be removed from the image by using wavelets. For noise removal Gabor filter is used. It is a linear filter all filters are generated from wavelet by performing dialation and rotation.

Pixel classification: It is a technique used in editing images. An image is blurred by displaying part or all of it at a clearly lower resolution. It is primarily used for censorship. The result is a standard graphics filter, vacant in all but the most basic bitmap graphics editors.

Image Segmentation: For image segmentation Thresholding is used. The image obtained after thresholding required less space for stoarage, it can process fastly and easy to conduct. Therefore, thresholding techniques have drawn a lot of awareness during the past 20 years. Thresholding is a non-linear action that converts a gray-scale image into a binary image where the two levels are assigned to pixels that are below or above the particular threshold value. In this research, Otsu's method is used. This method gives suitable results for bimodal histogram images. Threshold values based on this method will be between 0 and 1 , after achieving the threshold value; image will be segmented based on it.

Fuzzy logic: It is based on the mathematical theory of fuzzy sets, which is a simplification of the classical set theory. By introducing the notion of degree in the proof of a form, thus enabling a condition to be in a state other than true or false, it provides a very valuable elasticity for reasoning, which makes it possible to take into account inaccuracy and reservations. One advantage of fuzzy logic in order to formalize human reasoning is that the rules are set in normal language. In this detection method fuzzy logic is used for the conclusion. For the accurate conclusion apply the rules that is fuzzy rules.

Result analysis: The infected image with the disease matches with the given dataset. For the disease detection and classification, we are implementing the deep learning algorithm. algorithm is used to classify the specified image into appropriate disease hence it will be easy to detect the disease and find out the remedy over the disease. 


\section{Algorithm}

1. Input Image

2. Preprocess an Image

3. Classify Image Pixels into background, ROI and Infected Pixels.

4. Mark infected pixels.

5. Measures dimension of infected region i.e. height and width.

6. Perform Image segmentation so as to separate background, ROI and infected region.

7. Add infected region to database with proper naming.

8. Perform result analysis to get accurate conclusion.

9. Stop.

\section{Advantages}

1. To get a fracture detection system in which pathology admin will upload patient's scanned report.

2. To get an expert system for patients.

3. This system can provide quality medical care.

4. The use of this system can free the radiologist from the routine task.

5. It provides more accurate measurements that may lead to more cons.

\section{REFERENCES}

1. Chan, K.-P., Fu, A. W.-C. (1999). Efficient time series matching by wavelets. Data Engineering. In: Proceedings, 15th International Conference on, p. 126-133. IEEE.

2. Lim, S. E., Xing, Y., Chen, Y., Leow, W. K., Howe, T. S., Png, M. A. (2004). Detection of femur and radius fractures in X-ray images. In: Proc. 2nd Int. Conf. on Advances in Medical Signal and Info. Proc.

3. Yap, D. W.-H., Chen, Y., Leow, W. K., Howe, T. S., Png, M. A. (2004). Detecting femur fractures by texture analysis of trabeculae. In
Pattern Recognition. ICPR. In: Proceedings of the 17th International Conference on, 3, p. 730733. IEEE.

4. Lum, V. L. F., Leow, W. K., Chen, Y., Howe, T. S., Png, M. A. (2005). Combining classifiers for bone fracture detection in $\mathrm{x}$-ray images. In: Image Processing. ICIP. IEEE International Conference on, 1, p. I-1149. IEEE

5. Al-Khaffaf, H., Talib, A. Z., Salam, R. A. (2008). Removing salt-and-pepper noise from binary images of engineering drawings. In: Pattern Recognition. ICPR. 19th International Conference on, p. 1-4. IEEE.

6. Vijay Kumar, V., Vanathi, P., Kanagasabapathy, P. (2010). Fast and efficient algorithm to remove Gaussian noise in digital images. IAENG International Journal of Computer Science, 37(1).

7. Aishwariya, R., Geetha, M.Kalaiselvi. and Archana, M. "Computer- Aided Fracture Detection Of X-Ray Image”,

8. Anu T C, Mallikarjunaswamy M.S Rajesh Raman. Detection of Bone Fracture using Image Processing Methods. In International Journal of Computer Applications (0975 - 8887)

9. Swathika.B1,Anandhanarayanan.K,Baskaran.B, and Govindaraj.R.Radius Bone Fracture Detection Using Morphological Gradient Based Image Segmentation Technique.

10. Rathode, Hs. and Ali, Wahid. (2015), MRI Brain Image Quantification Using artificial neural networks - A Review Report, ISOI Journal of Engineering and Computer science, Vol. 1, Issue No. 1, pp. 48-55. (IJCSIT) International Journal of Computer Science and Information Technologies, Vol. 6 (2) , 2015, 1616-1619

11. Tanudeep Kaur, Anupam Garg. Bone Fraction Rathode, Hs. and Ali, Wahid. (2015), - MRI Brain Image Quantification Using artificial neural networks - A Review Report, International Journal of Engineering Trends and Technology (IJETT) - Volume 36 Number 2$\mathrm{Ju}$ e 2016 


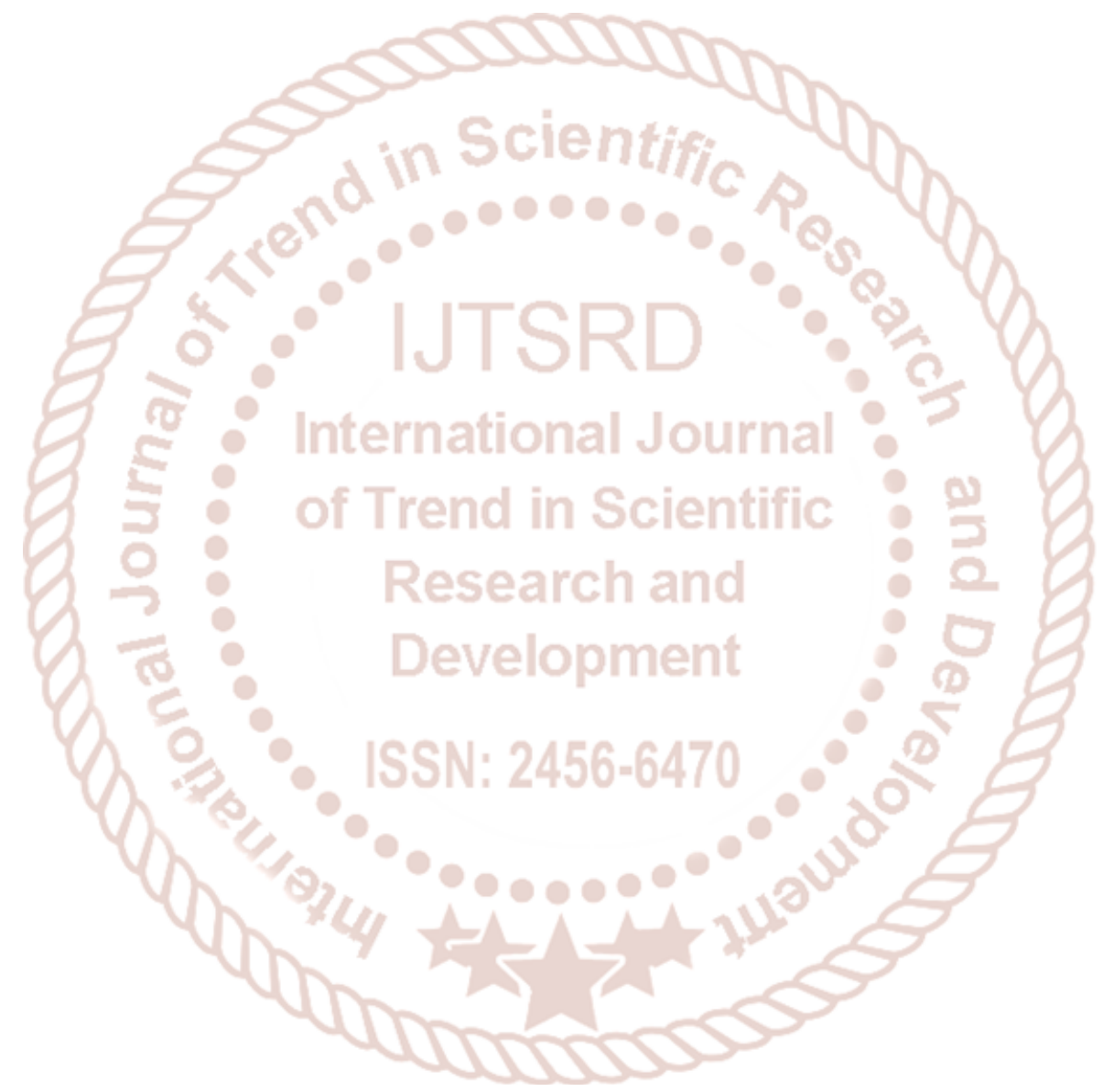

